\title{
New insights on the mode of action of microcystins in animal cells $-\mathbf{a}$ review
}

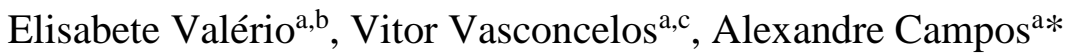

${ }^{a}$ Centro Interdisciplinar de Investigação Marinha e Ambiental, CIIMAR/CIMAR, Universidade do Porto, Rua dos Bragas 289, 4050-123 Porto, Portugal; ${ }^{b}$ Unidade de Água e Solo, Departamento de Saúde Ambiental, Instituto Nacional de Saúde Doutor Ricardo Jorge, Avenida Padre Cruz, 1649-016 Lisboa, Portugal; ' Departamento de Biologia, Faculdade de Ciências, Universidade do Porto, Rua do Campo Alegre, 4069-007 Porto, Portugal

\begin{abstract}
Microcystins (MCs) are the most commonly occurring hepatotoxins produced by cyanobacteria. The inhibition of PP2A is widely assumed as the principal mechanism of toxicity of MCs, however recently it has been found that MC modulates PP2A activity not only by direct inhibition of its activity, but also by regulating its expression. Nevertheless the mechanisms of toxicity of MCs seem to be more complex to interpret than expected. The induction of some cellularmolecular mechanisms appears to be biphasic in time and concentration of MC and in most cases related with the intracellular ROS generation. These intracellular ROS levels cause oxidative stress which leads to changes in several markers of MC-LR-induced oxidative stress ultimately resulting in apoptosis or cell damage and also genotoxicity. MCs can also induce severe changes in the cytoskeleton elements: microfilaments, intermediate filaments and microtubules, which results in changes in the cytoskeleton architecture and cell viability. There are also indications that there are second messengers involved in MC-LR mediated cytotoxicity and apoptosis. Different congeners of these toxins induce different degrees of responses in the cell, assumed to be related with the capacity of toxin internalization, affinity towards PP1 and PP2A, and the ability to cause oxidative stress. MCs have also been implicated in neurotoxicity and in damages in reproductive organs. The regulation of transcription factors and proto-oncogenes by MC is the mode of action of MCs tumor promotion. This review summarizes mainly the findings from the last five years about the molecular mechanisms behind MC toxicity in animal cells.
\end{abstract}

KEYWORDS: animal cells; apoptosis; cyanobacteria; microcystins; molecular mechanisms; oxidative stress

\footnotetext{
* Centro Interdisciplinar de Investigação Marinha e Ambiental, CIIMAR/CIMAR, Universidade do Porto. Rua dos Bragas, 289, P.O. Box: 4050-123, Porto, Portugal; Tel/Fax: +351- 223401800, +351-223390608; E-mail: acampos@ ciimar.up.pt
} 


\section{INTRODUCTION}

Microcystins (MCs) are toxic metabolites produced by several cyanobacterial (blue green algae) widely distributed in the environment. The genera usually associated with the production of these cyanotoxins are Microcystis, Anabaena, Nostoc, Planktothrix, Anabaenopsis, Hapalosiphon, and Nodularia. Over 80 structural congeners with a size between 909-1115 Da characterize this group of cyclic heptapeptides [1,2]. The general structure of MCs is cyclo(-D-Ala1-L-X2-D-erythro- $\beta$ methylAsp3-L-Z4-Adda5-D-Glu6-N-methyldehydro-Ala7) being $\mathrm{X}$ and $\mathrm{Z}$ variable L-amino acid residues that differentiate several of MC congeners and Adda the unique D-amino acid 3-amino-9-methoxy-2,6,8-trimethyl-10-phenyldeca-4,6-dienoic acid [3,4]. In addition amino acid modifications in the cyclic heptapeptide backbone characterize other MC congeners. Within this group the toxin congeners, MC-LR, -RR, and -YR are considered the most toxic and abundant species $[5,6]$.

MCs have received increasing attention in the past decades as a public health threat, to this awareness contributed the reports of human and animal intoxications and the increased evidences that MCs may cause serious health problems, such as various liver diseases, including primary liver cancer, hepatitis, and liver injury $[7,8]$. The oral intake is probably the major route of exposure to MCs in Humans. The tumor promoting potential of this toxin was related with the chronic exposure to low doses in drinking water [9].

The observations that MCs are potent inhibitors of serine/threonine-specific protein phosphatases (PPs), especially PP1 and PP2A [10-12], led to the widely accepted hypothesis that this molecular interaction is the main toxic mechanism of MCs. This mechanism is conserved among MC congeners, with different congeners showing comparable inhibitory capacity of PP1 and PP2A in vitro [13,14].

The toxicity of MCs is related with the rate of cell internalization. The molecular size render these molecules apparently incapable of crossing cell membranes via passive diffusion, therefore it is generally accepted that MCs enter the cells by active transport through specific transporters, the organic anion-transporting polypeptides (OATPs) $[15,16]$. It also has been found that MC-LR can produce a large amount of reactive oxygen species (ROS) in both in vitro and in vivo models as part of the toxicological action [17,18]. Following PP inhibition and oxidative stress a subsequent cascade of molecular events and signaling pathways are triggered resulting in lipid peroxidation, cytoskeletal disorganization, collapse of the cytoskeleton, increasing in levels of ROS, mitochondrial permeability transition, Cytochrome c release, and apoptosis [7]. The proteins p53, Bcl-2 and Bax were early associated to the regulation of MC-LR induced apoptosis [19].

However, the detailed toxicological mechanism involved in over-production of ROS remains unclear. Evidences suggest that the expression of p53, Bcl-2 and Bax are thought to be involved in the regulation of MC-LR induced apoptosis [19] but more functional studies are required to elucidate the exact mechanisms underlying the apoptosis-inducing potential of this toxin.

Novel evidences show the complex regulation of PP2A protein during the interaction with MC-LR, thereby leaving open the elucidation of the exact role of the protein in the toxic action of MCs in in vivo systems. Recent studies also have pointed out nitric oxide (NO), an important cellular secondary messenger influencing the development, progression and treatment of diseases, to participate in MC toxicity.

In this work we revised recent information of MCs toxicity molecular mechanisms in animal cells, using data from in vitro studies using e.g. cell lines, as well as, in vivo studies, namely using rats or fish, thereby providing an overview of the current state of the art in MC toxicity, which could facilitate the identification of novel and priority objectives of research. The genotoxicity of MCs is a well-studied topic which deserves a separate review and for this reason was not overviewed here.

\section{MODE OF ACTION OF MICROCYSTINS}

\subsection{Cellular uptake of MCs}

Microcystins (MCs) are molecules of relatively high molecular mass (900-1100 Da) [1], nevertheless their potent toxic action may denote a significant cellular internalization. Initial studies of cellular up-take with radiolabeled derivative of MC-LR have pointed out the cellular specificity of the transport in hepatocytes, moreover by means of the use of bile acid transporter inhibitors, the requirement of this active system for MC to enter the cells became evident [15]. The elucidation of the exact mechanism of MC transport, which involves different members of organic anion transporting polypeptides (OATPs), was later achieved with the Xenopus laevis oocyte expression system [20].

The specificity of this transport determines the main cellular targets of this group of toxins and likely the rate of uptake of the toxin. More recently Fisher and co-workers [21] demonstrated that HEK293 (Human Embryonic Kidney 293) cells stably expressing recombinant human OATP1B1/SLCO1B1 and OATP1B3/SLCO1B3 showed an increased susceptibility to MCs comparatively to control vector expressing cells. In this system and in primary hepatocytes, the cytotoxicity differences observed in the congeners MC-LW and -LF comparatively to MC-LR and MC-RR, were attributed to selective transport in the plasma membrane. Of notice is that all MC congeners show comparable PP-inhibiting capabilities. The proof of concept of the 
role of OATPs in MCs toxicity was also achieved with the assessment of cytotoxicity in cells differentially expressing members of this family of transporters. Cells expressing lower OATPs, like HepG2 (human liver carcinoma) and Jurkat T (T lymphocyte) cells, showed no sensitivity to MC-LR as compared to primary hepatocytes. However this response could be reverted with facilitated delivery of MC-LR (e.g. by inducing endocytosis) resulting in a marked enhancement of $\mathrm{HepG} 2 \mathrm{O}_{2}$ consumption and inhibition of Jurkat $\mathrm{T}_{2}$ consumption at concentrations above $0.1 \mathrm{nM}$ MC-LR. In this study the cellular respiration was the most sensitive test to low MC-LR, as compared to cell viability, total cellular ATP, extracellular acidification, ROS formation, or protein phosphorylation [22]. Following these results the authors proposed that MC-LR acts as an uncoupler of the Electron Transport Chain (ETC) affecting complex I functioning and increasing mitochondrial $\mathrm{O}_{2}$ consumption.

On the other hand the toxicity observed in cell types that do not express OATPs, such as epidermal cells, after prolonged incubation time with MCs [23], could be associated to a probable internalization by cell membrane diffusion of MCs.

\subsection{Inhibition of protein phosphatases}

Many cellular processes are regulated by reversible phosphorylation, a mechanism that is controlled by protein kinases and protein phosphatases, where they have opposing roles. Proteins are phosphorylated predominantly on Ser and Thr residues due to the activity of protein phosphatases types 1 (PP1) and 2A (PP2A). The discovery that MCs are potent inhibitors of PPs dates back to 1990 from the work of MacKintosh and co-workers that demonstrated in vitro inhibition of protein phosphatases 1 and 2A from both mammals and higher plants [24]. Since then the exact nature of interaction with PP1 and PP2A has been revealed, as well as the crystal structure of the complex. In synthesis, the interaction of MC-LR with PP1 and PP2A was reported as a two-step mechanism where the toxin first binds to the enzyme inactivating it and subsequently forms covalent adducts during prolonged reaction-time [24,25]. The interaction occurs between the hydrophobic groove, C-terminal groove and the catalytic site of PP-1c and the Glu, MeAsp and ADDA residues of the toxin [26]. The covalent binding with PP2A occurs between the $S \gamma$ atom of Cys 269 of the enzyme and the terminal carbon atom of the Mdha side chain of the toxin [27]. Early in vitro studies using the catalytic subunits of PP1 and PP2A enabled to determine IC50 values of $0.04 \mathrm{nM}$ for PP2A and $1.7 \mathrm{nM}$ for PP1 [28]. Moreover early studies pointed nuclear PP2A to be the primary target of MC-LR after acute exposure, followed by cytoplasmic PP2A [29]. Inhibition of these enzymes disrupts the balance between the states of phosphorylation and dephosphorylation, leading to hyperphosphorylation of proteins involved in the dynamic organization of the cytoskeleton which, in turn, induces changes in the structure of hepatocytes.

More recent evidences suggest that MC modulates PP2A activity not only by direct inhibition of PP2A activity, but also by regulating its expression. Some studies using human cellular lines (human amniotic epithelial (FL) cells) and human hepatoma cells (Huh7) show that MC-LR increases PP2A phosphatase activity through upregulating the mRNA and protein levels of PP2A [30,31]. When analyzing the effects of MCs on phosphoprotein phosphatases gene expression in the freshwater clam Corbicula fluminea, it was verified that there is a significant induction of PP2 gene expression, opposing to the lack of changes in the PP1 and PP4 gene expression [32]. Hence there is the possibility that organisms respond to PP2A inhibition by synthesizing more enzyme to replace the activity.

Despite the advances in the elucidation of MC mediated PP1A and PP2A inhibition, the mechanism is not fully disclosed. Indeed PP2A are holoenzymes composed of a dimeric core with structural A (PP2A/A) and catalytic C (PP2A/C) subunits which are regulated by several regulatory B subunits and by post-translational modifications (PTMs). Each of the A and C subunits are coded by two genes in mammalian cells, whereas B subunits are coded by four subfamilies of genes, hence conferring PP2A distinct functions and characteristics [27,33]. This opens the hypothesis of a possible differential affinity of MCs to the holoenzymes determined by protein sequence, PTMs and the functions of the variable regulatory subunits. In this respect the interaction of MCs to the PP2A holoenzyme could define a new mode of interpretation of its toxicity. MC-LR has shown to induce a dual response in PP2A. At low concentration the toxin stimulates, rather than inhibits, PP2A activity of human embryonic kidney HEK293 cells [34]. Immunoprecipitation and immunofluorescence assays performed by the researchers revealed that the catalytic subunit and a regulatory subunit of PP2A, termed $\alpha 4$, dissociate from inactive complex upon MC-LR exposure, which could have originated the gain of activity of the catalytic subunit in the cellular inactive protein complex pool [34]. This mechanism was suggested to compensate the inhibition induced by MC-LR. However at high concentration the toxin decreases PP2A activity of HEK293 cells, as a result of a superior inhibition reaction to the compensatory effect. PP2A inhibition was followed by the destabilization of HEK293 cells cytoskeleton architecture, detachment to extracellular matrix and further anoikis [34]. The results place $\alpha 4$ not only in the heart of PP2A activity regulation but also in mediation of toxicity of MCs. The second messenger ceramide has also been referred to intervene in this function (mechanism detailed in section 2.4). 


\subsection{Oxidative stress}

Oxidative stress can be defined as the exposure of the cell or tissue to an excess level of oxidants, particularly to free radicals, such as peroxide, superoxide or hydroxyl radicals, which are usually referred as reactive oxygen species (ROS). Oxidative stress occurs either due to an overproduction of ROS or to a decrease of the cellular antioxidant levels [35]. Oxidative stress has been regarded, in parallel with PP2A inhibition, as one of the major pathways of MCs cytotoxicity. MC-LR mediated oxidative stress was first reported in primary cultures of rat hepatocytes [36] and subsequently verified in other in vitro systems [37-39] and in vivo [40-42]. Previous studies indicated that oxidative damage is dose-dependent [36,40] and is characterized by alterations in several markers of cytotoxicity (lactate dehydrogenase leakage), lipid peroxidation (e.g. malondialdehyde), ROS and antioxidant enzymes [35,43]. Oxidative stress is intrinsically related with mitochondria metabolism and can conduct to cell death by necrosis or apoptosis and to genotoxicity. Earlier evidences led to the suggestion that ROS formation is a consequence of mitochondrial permeability transition (MPT) and loss of mitochondrial membrane potential (MMP) mediated by cellular $\mathrm{Ca}^{2+}$ levels $[44,45]$. MPT subsequently triggers the release of apoptotic factors from mitochondria, such as cytochrome c, Bax and Bid proteins and leads to cell death and cytotoxicity [41,44].

In the mitochondria-dependent apoptotic pathway the c-Jun N-terminal protein kinase (JNK) plays an important regulatory role. The earlier findings showed that MC-LR induces the activation of JNK [46]. This event affects some crucial enzymes of energy metabolism and leads to mitochondria dysfunction induced by MC-LR. Activity of JNK may, in this way, contribute significantly to hepatocyte apoptosis and oxidative liver injury by MC-LR [46].

Recent studies have once again pointed out the increase of intracellular ROS as a main marker of exposure to MC-LR [47,48]. ROS generation has biphasic time, is toxin-concentration dependent [49] and is related to impairment of mitochondrial respiratory chain and oxidative phosphorylation system [50]. It was also recently shown that the ability to cause oxidative stress may underlie the differential toxicity observed between chemical variants, MC-LR and MC-RR. The toxic action of the two variants in intestinal cells was linked to oxidative stress and to the expression of pro-inflammatory IL-6 and IL-8 proteins, IL-8 accumulation being more dependent on the MC variant [51]. As to shed more light on the role of oxidative stress on the toxicity induced by MCs, this biochemical process was investigated with and without the effect of the antioxidants $\mathrm{N}$-acetyl-cysteine (NAC) and buthionine sulfoximine (BSO), in the liver of the model fish Cyprinus carpio L. The action of MC-LR is followed by a series of molecular events linked with oxidative stress, such as the increase of hydroxyl radical $(\bullet \mathrm{OH})$ and the subsequent induced expression of apoptosis-related genes, including $\mathrm{p} 38$, JNKa, and bcl-2. Strikingly NAC provided significant protection to the cytoskeleton, thereby evidencing that oxidative stress is a key process in the mode of action of MCs and that the toxicological outcomes could be partially counteracted by protecting the tissues from oxidative stress [52].

In the same line of research the role of the nuclear factor erythroid 2-related factor 2 (Nrf2) on the oxidative stress mediated MC-LA toxicity was investigated in wild type (WT), Nrf2-null and Keap1-HKO knock-down mice [53]. Nrf2 has been considered an important defense mechanism to oxidative and electrophilic stresses in the liver, by regulating the expression of a conjunct of antioxidant proteins $[54,55]$. In the cell Nrf2 is regulated by degradation via ubiquitin system and involving the participation of Kelch like-ECH-associated protein 1 (Keap1) and Cullin 3 [54,55]. Under oxidative/electrophilic stress Keap1 activity is disrupted, stopping the degradation of Nrf2. The transcription factor will then activate the oxidative defense system. $\mathrm{Lu}$ and co-workers [53] demonstrated that Nrf2 is also involved in MC-LA toxicity and mediates the cellular action of the toxin. The authors reported an increase of Nrf2 and Nqo1 mRNAs and proteins in Keap1-HKO knock-down mice at constitutive levels and after MC challenge. Moreover MC-LA markedly depleted liver GSH by 60-70\% in Nrf2-null and WT mice but only 35\% in Keap1-HKO knock-down mice. The mRNAs of GSH conjugation and peroxide reduction enzymes, such as Gsta1, Gsta4, Gstm, and Gpx2 were higher in livers of Keap1-HKO mice, together with higher expression of the ratelimiting enzyme for GSH synthesis (Gclc) [53].

\subsection{Cytotoxicity}

There are three cytoskeleton structural elements: microfilaments (MFs), intermediate filaments (IF) and microtubules (MT). It had been previously demonstrated that the exposure to MC induces several cystoskeletal alterations, particularly the hyperphosphorylation of cystoskeletal proteins, such as cytokeratins, through PP1 and PP2A inhibition which leads to reorganization, disassembly, and consequently to disruption of cellular architecture [11,56]. Gácsi et al. [57] tested MC-LR toxic effects in a concentration and time dependent manner and verified apoptotic shrinkage in cells treated with 5-50 $\mu \mathrm{M} \mathrm{MC-}$ LR for $24 \mathrm{~h}$ which displayed a reduction on the number of cells presenting normal actin, the degradation of microfilament mesh, and a concentration dependent depolymerization of microtubules [57]. Also, the inhibition of PP2A could lead to the activation of mitogen-activated protein kinases (MAPKs) including ERK1/2, JNK, and p38, which subsequently participate to apoptosis [58].

More recently, Liang et al. [30] observed that exposure to MC-LR impairs the ability of B55 $\alpha$-containing PP2A to bind to tyrosinated tubulin, and suggested that PP2A is involved in the MT destabilization induced by MC-LR. Moreover, Huang et al. [48] showed aggregation and collapse of microfilaments (MFs) and microtubules (MTs) in CIK (Ctenopharyngodon idellus kidney) cells exposed to MC-LR and also loss of some cytoskeleton structure. The authors also observed transcriptional changes of cytoskeletal genes ( $\beta$-actin, lc3a, and keratin), that are probably related with cytoskeleton structure damage [48]. 
These findings raised the hypothesis that oxidative stress and cytoskeletal disruption are interconnected and both contribute to apoptosis and renal toxicity induced by MCs [48]. Furthermore, mRNA and protein levels of microfilament associated proteins ezrin, vasodilator stimulated phosphoprotein (VASP), actin-related protein (ARP) $2 / 3$ and cofilin remained unchanged in normal human liver HL7702 cells [59]. However, the actin filaments lost their characteristic filamentous organization after treatment with MC-LR, revealing increased actin depolymerization [59]. Major IF proteins, such as keratin 18, vimentin and lamin A/C maintain their mRNA and protein levels after exposure to MC-LR, despite the accumulation of IFs around the nucleus, which led to the formation of dense bundles [60]. MC-LR doses 10 times above the guideline value $\left(1 \mu \mathrm{g} \mathrm{L^{-1 }}\right)$ led to a decrease of cell proliferation and an increase in apoptosis and cell cycle arrest [60].

Indeed MCs can induce apoptosis in a variety of cell types. Apoptosis is a mechanism used by cells and tissues in response to various toxic compounds, characterized by distinct morphological features such as cell shrinkage, condensation of nuclear chromatin and of the cytoplasm, detachment of the cells from surrounding cells, plasma membrane blebbing, oligonucleosomal DNA fragmentation, externalization of membrane phosphatidylserine and ultimately the breakdown of the cell into smaller units (apoptotic bodies) [35,61]. Apoptosis may be induced by both intrinsic and extrinsic pathways. The extrinsic pathway occurs when appropriate exogenous mediators or ligands bind to pro-apoptosis receptors of the target cells to induce apoptosis. Fas ligand (FasL) and Fas receptor (Fas) signaling is one of the major extrinsic pathways initiating apoptosis [61]. The mechanisms behind the apoptosis induction by MCs have been under investigation. Feng et al. [61] showed that MC-LR induced Fas receptor (Fas) and Fas ligand (FasL) expression, at both mRNA and protein levels system, are mediated by nuclear factor kappa-light-chain-enhancer of activated B cells (NF- $\mathrm{kB})$, a known protein complex that functions as a transcription factor and plays a fundamental role in the immune response to infection and is involved in the response to several other cellular stimuli [62], thus leading to cellular apoptosis in HepG2 cells. In agreement with this the severe stress effects and apoptosis in the rat insulinoma cell line INS-1 upon chronic exposure to MC were shown to be correlated with the selective activation of NF-kB [63].

There are also evidences that MCLR exerts its hepatotoxicity through MAPK pathway activation, leading to the hyperphosphorylation of IF proteins, namely p38, ezrin and ERK1/2 in a concentration-dependent manner [59,60]. The activation of p38-MAPK revealed to be biphasic in time and concentration and related with the intracellular ROS generation [49]. This mechanism resulted in changes in the cytoskeleton architecture and cell viability.

It has also been shown that MC variants -LF and -LW, which are more hydrophobic than MC-LR, had more pronounced cytotoxic effects on Caco-2 (human colon adenocarcinoma) as compared to MC-LR [64]. Which factors determine the differential toxicity of these MC variants need elucidation, nevertheless could be related with the capacity of toxin internalization, affinity towards PP1 and PP2A, and the ability to cause oxidative stress.

Cytotoxic effects have also been observed in immune cells. Rymuszka and Adaszek [65] verified that lower doses of MC-LR induced apoptosis in lymphocytes, whereas high toxin concentrations induce necrosis in lymphocytes in a time- and concentration-dependent manner. Moreover, the authors observed a re-organization of the actin cytoskeleton in phagocytes, followed by cell shrinkage and the disappearance of filopodia. Based on these observations authors suggested that both phagocytes and lymphocytes are also MC-LR targets and that disturbances of phagocytosis may impair the balance of the immune system [65].

The health consequences of the epidermal exposure to MC-LR have been less investigated. However this route of exposure could be of concern particularly in cases of skin damage (injury, sunburn) that would enable faster penetration of the toxin, leading to contact with the deeper cell layers. To this regard the impact of MC-LR on processes indispensable for normal skin function and regeneration, namely, viability, migration and actin cytoskeleton organization of human keratinocytes was examined. The results showed that short exposure to MC-LR does not affect proliferation of human skin keratinocytes but it is toxic after longer incubation in dose dependent manner. The observed effects ranged from the inhibition of keratinocyte migration, cell proliferation, cell enlargement, depolymerization of the F-actin cytoskeleton [23].

\subsection{Second messengers}

As described above calcium was one of the first second messengers to be discovered in the context of MC toxicity. Calcium may trigger MC cell death via mitochondria [41,44,45]. However this might not be the only intracellular signaling molecule participating in signal transduction during MC exposure. When Krakstad et al. [66] were trying to address the role of calcium calmodulin-dependent multifunctional protein kinase II (CaMKII) in MC induced hepatocytes apoptosis, they verified that antioxidants ( $\mathrm{N}$-acetylcysteine, Tempol or naringin), prevented chromatin condensation when given after the CaMKIIdependent commitment point. The authors suggested that CaMKII had mediated the accumulation of a second messenger belonging to reactive oxygen species [66].

$\mathrm{Li}$ and coworkers [67] investigated the hypothesis of the involvement of the second messenger ceramide in MC-LR mediated cytotoxicity. Ceramide is an important lipid-like second messenger generated by cells and is involved in many cellular processes, including PP2A activation, cytoskeleton destabilization and apoptosis [68,69]. The population of PP2A being 
activated by ceramide is termed ceramide-activated protein phosphatase (CAPP) [70]. CAPP consists of PP2A AC (dimeric) or PP2A AC enzymes associated to the regulatory subunits B55 $\alpha$ or B56 $\alpha$ (trimeric enzymes, PP2A ABC) [71].

The MC-LR induced toxicity in vivo and in vitro correlated with the generation of ceramide and with other cellular effects including inhibition of PP2A, regulation of PP2A subunit protein levels and subcellular localization of PP2A/B55 $\alpha$, contraction of the Golgi apparatus, cytoskeleton destabilization and cell detachment [67]. The relevance of ceramide in MC-LR toxicity was further evidenced with the utilization of ceramide synthase inhibitor, desipramine, which leads to protection of cells from the toxin and cytoskeleton destabilization (polymerization of actin filaments, contraction of microtubulin and vimentin), cell detachment, and apoptosis induced by MC-LR [67]. The effects of desipramine in PP2A activity pointed out for a central role of ceramide in PP2A regulation, and for the putative involvement of the second messenger in the PP2A activity stimulation or inhibition reported in respectively non-toxic and toxic MC-LR concentrations [34,67].

Moreover NF-kB, the protein complex that controls transcription of DNA, cytokine production and cell survival, was shown to be activated in MC-LR treated apoptotic HepG2 cells [61]. Following NF-kB activation there is an increase of mRNA and protein levels of nitric oxide synthase (iNOS). This mechanism is hypothesized to stimulate nitric oxide (NO) production from iNOS and to trigger apoptosis in a dose dependent manner [63]. Since the abrogation of NF-kB resulted in the reduction of apoptosis it is suggested that NF-kB/iNO signaling pathway play a major role in MC-LR mediated apoptosis [63]. This second messenger has been also related with the genotoxic effects in the chronic MC-LR exposure of human-hamster hybrid cells [72].

\subsection{Neurotoxicity}

The neurotoxic action of MC is relatively less characterized compared with the genotoxicity. For this reason it is a mechanism less described. There are evidences that MC-LR is also brain-permeable [20,73] thus presenting the possibility that MC-LR could exert neurotoxicity. The mechanisms underlying neurotoxicity have been object of some studies. It was observed that MC-LR induced hyperphosphorylation of neural microtubule-associated protein tau in a biphasic time and concentration dependent manner which correlated with an increase in soluble tau and a decrease in cytoskeleton-associated tau [49,74]. Tau proteins are low-molecular-weight microtubule-associated proteins that are abundant in nerve cells. When tau proteins are phosphorylated they induce the destabilization of microtubules, compromise axonal transport, and contribute to neuronal degeneration [75]. When Meng et al. [74] tried to determine if tau was involved in MC-LR induced reorganization and destabilization of the MT structure, they used the Tau-1 antibody, which specifically recognizes the dephosphorylated form of tau containing Ser-195, Ser-198, Ser-199, Ser-202 and Thr-205 epitopes. They observed that exposure to MC-LR resulted in a decrease in the Tau-1 recognized epitope, especially at $10 \mu \mathrm{M}$, where there was a severe PP2A inhibition, suggesting that MCLR reduced the dephosphorylation of tau [74]. This suggestion was confirmed when the authors used anti-phospho-tau (pSer199/202) antibody, to directly recognize the phosphorylated forms of tau at Ser-199 and Ser-202, and detected a progressively enhanced tau phosphorylation in response to increasing MC-LR concentrations [74]. Furthermore, to determine whether ROS was involved in MC-LR tau hyperphosphorylation the action of the antioxidants, N-acetylcysteine (NAC) and vitamin $C$ was tested [49]. It was verified that the phosphorylation level of tau was effectively recovered due to the inhibition of ROS production [49]. Therefore it was suggested that simultaneous alteration of tau phosphorylation at the Ser-199 and Ser-202 residues, is closely related to previous ROS generation [49,74].

Li et al. [76] have shown that MC-LR is accumulated in the hippocampi of Wistar rats upon intraperitoneal injection of the toxin. This is followed by impairment of neurological functions assessed with behavioral testing. The mechanisms underlying the neurotoxicity of MC-LR may initiate with the inhibition of serine/threonine protein phosphatases (PPs) and hyperphosphorylation of proteins such as the neuronal microtubule-associated protein tau and the differential expression of septin 5, $\alpha$-internexin, and $\alpha$-synuclein. However global protein profiling demonstrated that MC-LR produce significant alterations at the proteome level, affecting proteins involved in cytoskeleton, neurodegenerative disease, oxidative stress, apoptosis, and energy metabolism [76].

\subsection{Effects on reproductive organs}

Several recent studies have been showing that MC-LR also exerts toxic effects in reproductive system. The first evidences came from the studies from Ding et al. [77] where the reproductive toxicity of Microcystis aeruginosa cell extracts containing MCs was tested on male mice. The authors verified a number of alterations on mice testis and epididymides. Later Li et al. [78] observed cytotoxicity, oxidative stress and apoptosis of Leydig cells which resulted in decreased testosterone production and consequently in reproductive toxicity.

Overall, the reproductive toxicity is manifested by reduced testosterone levels, testicular atrophy, low sperm motility, and a high incidence of sperm abnormalities in a dose- and time-dependent manner [50]. Moreover testis impairment was related to ROS and oxidative phosphorylation, cytoskeleton depolymerization and mitochondria dysfunction [79]. The viability of Sertoli cells, which play an essential role in the development and maturation of sperm cells, was diminished after treatment with MCLR, which induced apoptosis and autophagy [50,80]. Results show that the apoptosis is likely mediated by the expression of the transcription factor p53, and by the apoptotic regulators bax, bcl-2 and caspase-3 [81]. Furthermore, Zhou et al. [81] showed that MC-LR can increase the number of apoptotic testicular cells, decrease cellular proliferation and disrupt histophatological 
structures of spermatogenic cells in vivo. Structural changes in testis, tubular diameter and relative weight of testes were also decreased.

The reproductive toxicity induced by MC-LR was also assessed on female mice [82]. Here a reduction of ovary weight was observed and attributed to loss of primordial follicle pool provoked by MC-LR exposure. It was also verified that MC-LR could accumulate in ovary and directly induce reproductive toxicity [82].

\subsection{Tumor promotion}

Humpage and Falconer [83] have taken the first steps towards the understanding of the mechanisms of the tumor promotion activity of MC that was speculated from exposure evidences. Based on these studies and the collected evidences of the tumor promoting activity, MC-LR - the most abundant and toxic microcystin congener - was classified by International Agency for Research on Cancer (IARC) as a potential carcinogen to humans (class 2B) [84].

With the studies of Clark et al. [85] the role of p53 in MC-LR induced tumor promotion became evident. Furthermore evidences were collected using the monkey kidney-derived cell line Vero-E6 which suggest the involvement of ERK1/2 pathway [86]. Li et al. [87] also observed that the exposure to MC extracts greatly induced the expression of proto-oncogenes c-fos, c-jun and c-myc in mouse liver, kidney and testis, making it a possible mechanism for MC tumor-promoting activity. Recently Fan et al. [88] suggested that hepatic tumorigenesis may also be related with MC-LR stimulation of proto-oncogene cmyc transcription and the inhibition of protein phosphatase PP2A activity, which has a stabilization effect on c-myc protein, by altering the phosphorylation status of serine 62. The higher susceptibility of liver to MCs may be in part related with the OATP transport system highly abundant in hepatocytes thereby favoring the uptake of the molecule and its tumor promotion activity.

The tumorigenic action of MC-LR was suggested to be also under the regulation of the transcription factor NF- $\mathrm{KB}$, and the subsequent induction of the expression of interferon alpha (IFN- $\alpha$ ) and tumor necrosis factor alpha (TNF- $\alpha$ ) [31].

\subsection{Global changes in protein expression}

The global gene/protein analysis strategies offer a unique opportunity to report and integrate the multiple molecular events occurring in the cells. They are therefore essential strategies to investigate and to disclose the mode of action of environmental contaminants such as algal toxins and MCs. In this context a proteomics strategy have led to the observation that MC-RR activity in human amniotic epithelial cells (FL) induces several early proteomic alterations related with signal transduction, gene transcription and translation, and post-immediate alterations related with cell metabolism, apoptosis, protein degradation, cell cycle, cell differentiation, transport functions [89]. Within the differentially expressed proteins Hsp 70 is a known determinant of cell death and key regulator of multiple cellular processes. Evidences also confirm the role of p53 and its potential targets in MC mediated response and provide first indication that PP2A/A subunit is up-regulated while a number of proteins implicated in the ubiquitin-proteasome pathway are down-regulated in cells exposed to MC-RR [89]. The authors advance the hypothesis that MCs may modulate PP2A activity through regulation of its expression rather than inhibition of its activity directly.

\section{CONCLUSION}

MCs are potent toxins that induce major damages in animal cells through cytotoxic and genotoxic effects leading to apoptosis, tumor promotion and cancer as summarized in Figure 1. More recently, it has also been verified that MC is also able to induce neurotoxic effects, and provoke damages in reproductive organs. The molecular mechanisms underlying the toxicity of MC seems to be a network of causal-effect cascades that are interconnected and lead to a final outcome of damage to the cell and apoptosis. The main mechanism of action is possibly triggered by the inhibition of PP2A, which in combination with the increase of the intracellular ROS levels induced by MC leads to a series of cellular alterations and damages. In Figure 1 we summarize the main so far described effects induced by MCs on animal cells.

Beyond studying the effect and consequences of exposure to MCs, there have been also some efforts in searching possible ways to minimize and remediate the disease effects caused by cyanotoxins. The use of antioxidants such as N-acetylcysteine (NAC), buthionine sulfoximine (BSO) and vitamin $\mathrm{C}$ can contribute to attenuate the toxic effects induced by MC. Furthermore, chemotherapeutical strategies, leading to 553 inactivation, are also a possibility to overcome MCs poisoning [49,90]. 


\section{CONFLICT OF INTEREST}

The authors have nothing to disclose.

\section{ACKNOWLEDGEMENTS}

This research was partially supported by the Strategic Funding UID/Multi/04423/2013 through national funds provided by FCT - Foundation for Science and Technology and European Regional Development Fund (ERDF), in the framework of the programme PT2020 and through Post-Doc research grants from FCT to E. Valério (SFRH/BPD/75922/2011) and A. Campos (SFRH/BPD/103683/2014).

\section{REFERENCES}

[1] Sivonen, K.; Jones, G. Cyanobacterial toxins. In Toxic cyanobacteria in water - a guide to their public health consequences, monitoring and management; Chorus, I., Bartram, J., Eds.; WHO, E \& FN Spon: London, UK, 1999; pp 41-111.

[2] Humpage, A. Toxin types, toxicokinetics and toxicodynamics. In Cyanobacterial Harmful Algal Blooms: State of the Science and Research Needs; Hudnell, H. K., Ed.; Springer New York, 2008; pp 383-415.

[3] Botes, D.P.; Tuinman, A.A.; Wessels, P.L.; Viljoen, C.C.; Kruger, H.; Williams, D.H.; Santikarn, S.; Smith, R.J.; Hammond, S.J. The structure of cyanoginosin-LA, a cyclic heptapeptide toxin from the cyanobacterium Microcystis aeruginosa. J. Chem. Soc. Perkin Trans. 1, 1984, 0, $2311-2318$.

[4] Botes, D.P.; Wessels, P.L.; Kruger, H.; Runnegar, M.T.C.; Santikarn, S.; Smith, R.J.; Barna, J.C.J.; Williams, D.H. Structural studies on cyanoginosins-LR, -YR, -YA, and -YM, peptide toxins from Microcystis aeruginosa. J. Chem. Soc. Perkin Trans. 1, 1985, 0, $2747-2748$.

[5] Carmichael, W.W. The toxins of cyanobacteria. Sci. Am., 1994, 270 (1), 78-86.

[6] Dawson, R.M. the toxicology of microcystins. Toxicon, 1998, 36 (7), 953-962.

[7] Dittmann, E.; Wiegand, C. Cyanobacterial toxins - occurrence, biosynthesis and impact on human affairs. Mol. Nutr. Food Res., 2006,50 (1), 7-17.

[8] Van Apeldoorn, M.E.; van Egmond, H.P.; Speijers, G.J.A.; Bakker, G.J.I. Toxins of cyanobacteria. Mol. Nutr. Food Res., 2007,51 (1), 7-60.

[9] Nishiwaki-Matsushima, R.; Ohta, T.; Nishiwaki, S.; Suganuma, M.; Kohyama, K.; Ishikawa, T.; Carmichael, W.; Fujiki, H. Liver tumor promotion by the cyanobacterial cyclic peptide toxin microcystin-LR. J. Cancer Res. Clin. Oncol., 1992, 118 (6), 420-424.

[10] Runnegar, M.; Berndt, N.; Kong, S.M.; Lee, E.Y.C.; Zhang, L.F. In vivo and in vitro binding of microcystin to protein phosphatase 1 and $2 \mathrm{~A}$. Biochem. Biophys. Res. Commun., 1995, 216 (1), 162-169.

[11] Toivola, D.M.; Goldman, R.D.; Garrod, D.R.; Eriksson, J.E. Protein phosphatases maintain the organization and structural interactions of hepatic keratin intermediate filaments. J. Cell Sci., 1997, 110 (1), 23-33.

[12] Hastie, C.J.; Borthwick, E.B.; Morrison, L.F.; Codd, G.A.; Cohen, P.T.W. Inhibition of several protein phosphatases by a non-covalently interacting microcystin and a novel cyanobacterial peptide, nostocyclin. Biochim. Biophys. Acta - Gen. Subj., 2005, 1726 (2), 187-193.

[13] Robillot, C.; Hennion, M.C. Issues arising when interpreting the results of the protein phosphatase 2A inhibition assay for the monitoring of microcystins. Anal. Chim. Acta, 2004, 512 (2), 339-346.

[14] Hoeger, S.J.; Schmid, D.; Blom, J.F.; Ernst, B.; Dietrich, D.R. Analytical and functional characterization of microcystins for risk assessment? Environ. Sci. Technol., 2007, 41 (7), 2609-2616.

[15] Eriksson, J.E.; Grönberg, L.; Nygård, S.; Slotte, J.P.; Meriluoto, J.A.O. Hepatocellular uptake of ${ }^{3}$ H-dihydromicrocystin-LR, a cyclic peptide toxin. Biochim. Biophys. Acta, 1990, 1025 (1), 60-66.

[16] Runnegar, M.; Berndt, N.; Kaplowitz, N. Microcystin uptake and inhibition of protein phosphatases: effects of chemoprotectants and self-inhibition in relation to known hepatic transporters. Toxicol. Appl. Pharmacol., 1995, 134 (2), 264-272.

[17] Ding, W.X.; Shen, H.M.; Ong, C.N. Critical role of reactive oxygen species and mitochondrial permeability transition in microcystin-induced rapid apoptosis in rat hepatocytes. Hepatology, 2000, 32 (3), 547-555.

[18] Bouaïcha, N.; Maatouk, I. Microcystin-LR and nodularin induce intracellular glutathione alteration, reactive oxygen species production and lipid peroxidation in primary cultured rat hepatocytes. Toxicol. Lett., 2004, 148 (1-2), 53-63.

[19] Brzuzan, P.; Woźny, M.; Ciesielski, S.; Łuczyński, M.K.; Góra, M.; Kuźmiński, H.; Dobosz, S. Microcystin-LR induced apoptosis and mRNA expression of p53 and cdkn1a in liver of whitefish (Coregonus lavaretus L.). Toxicon, 2009, 54 (2), 170-183.

[20] Fischer, W.J.; Altheimer, S.; Cattori, V.; Meier, P.J.; Dietrich, D.R.; Hagenbuch, B. Organic anion transporting polypeptides expressed in liver and brain mediate uptake of microcystin. Toxicol. Appl. Pharmacol., 2005, 203 (3 SPEC. ISS.), 257-263.

[21] Fischer, A.; Hoeger, S.J.; Stemmer, K.; Feurstein, D.J.; Knobeloch, D.; Nussler, A.; Dietrich, D.R. The role of organic anion transporting polypeptides (OATPs/SLCOs) in the toxicity of different microcystin congeners in vitro: A comparison of primary human hepatocytes and OATPtransfected HEK293 cells. Toxicol. Appl. Pharmacol., 2010, 245 (1), 9-20.

[22] Jasionek, G.; Zhdanov, A.; Davenport, J.; Blaha, L.; Papkovsky, D.B. Mitochondrial toxicity of microcystin-LR on cultured cells: application to the analysis of contaminated water samples. Env. Sci, Technol., 2010, 44 (7), 2535-2541. 
[23] Kozdęba, M.; Borowczyk, J.; Zimoląg, E.; Wasylewski, M.; Dziga, D.; Madeja, Z.; Drukala, J. Microcystin-LR affects properties of human epidermal skin cells crucial for regenerative processes. Toxicon, 2014, 80, 38-46.

[24] MacKintosh, C.; Beattie, K.A; Klumpp, S.; Cohen, P.; Codd, G.A. Cyanobacterial microcystin-LR is a potent and specific inhibitor of protein phosphatases 1 and 2A from both mammals and higher plants. FEBS Lett., 1990, 264 (2), 187-192.

[25] Craig, M.; Luu, H.A.; McCready, T.L.; Williams, D.; Andersen, R.J.; Holmes, C.F.B. Molecular mechanisms underlying the interaction of motuporin and microycystins with type-1 and type-2A protein phosphatases. Biochem. Cell Biol. Biol. Cell., 1996, 74 (4), 569-578.

[26] Goldberg, J.; Huang, H.B.; Kwon, Y.G.; Greengard, P.; Nairn, A.C.; Kuriyan, J. Three-dimensional structure of the catalytic subunit of protein serine/threonine phosphatase-1. Nature, 1995, 376 (6543), 745-753.

[27] Xing, Y.; Xu, Y.; Chen, Y.; Jeffrey, P.D.; Chao, Y.; Lin, Z.; Li, Z.; Strack, S.; Stock, J.B.; Shi, Y. Structure of protein phosphatase 2A core enzyme bound to tumor-inducing toxins. Cell, 2006, 127 (2), 341-353.

[28] Honkanen, R.E.; Zwiller, J.; Moore, R.E.; Daily, S.L.; Khatra, B.S.; Dukelow, M.; Boynton, A.L. Characterization of microcystin-LR, a potent inhibitor of type 1 and type 2A protein phosphatases. J. Biol. Chem., 1990, 265 (32), 19401-19404.

[29] Billam, M.; Mukhi, S.; Tang, L.; Gao, W.; Wang, J.-S. Toxic response indicators of microcystin-LR in F344 rats following a single-dose treatment. Toxicon, 2008, 51 (6), 1068-1080.

[30] Liang, J.; Li, T.; Zhang, Y.-L.; Guo, Z.-L.; Xu, L.-H. Effect of microcystin-LR on protein phosphatase 2A and its function in human amniotic epithelial cells. J. Zhejiang Univ. Sci. B, 2011, 12 (12), 951-960.

[31] Christen, V.; Meili, N.; Fent, K. Microcystin-LR induces endoplasmatic reticulum stress and leads to induction of NF- $\kappa B$, interferon-alpha, and tumor necrosis factor-alpha. Environ. Sci. Technol., 2013, 47 (7), 3378-3385.

[32] Martins, J.C.; Machado, J.; Martins, A.; Azevedo, J.; OlivaTeles, L.; Vasconcelos, V. Dynamics of protein phosphatase gene expression in Corbicula fluminea exposed to microcystin-LR and to toxic Microcystis aeruginosa cells. Int. J. Mol. Sci., 2011, 12 (12), $9172-9188$.

[33] Xu, Y.; Xing, Y.; Chen, Y.; Chao, Y.; Lin, Z.; Fan, E.; Yu, J.W.; Strack, S.; Jeffrey, P.D.; Shi, Y. Structure of the protein phosphatase 2A holoenzyme. Cell, 2006, 127 (6), 1239-1251.

[34] Li, T.; Huang, P.; Liang, J.; Fu, W.; Guo, Z.; Xu, L. Microcystin-LR (MCLR) induces a compensation of PP2A activity mediated by $\alpha 4$ protein in HEK293 cells. Int. J. Biol. Sci., 2011, 7 (6), 740-752.

[35] Ding, W.-X.; Nam Ong, C. Role of oxidative stress and mitochondrial changes in cyanobacteria-induced apoptosis and hepatotoxicity. FEMS Microbiol. Lett., 2003, 220 (1), 1-7.

[36] Ding, W.X.; Shen, H. M.; Zhu, H.G.; Ong, C.N. Studies on oxidative damage induced by cyanobacteria extract in primary cultured rat hepatocytes. Environ. Res., 1998, 78 (1), 12-18.

[37] Nong, Q.; Komatsu, M.; Izumo, K.; Indo, H.P.; Xu, B.; Aoyama, K.; Majima, H.J.; Horiuchi, M.; Morimoto, K.; Takeuchi, T. Involvement of reactive oxygen species in Microcystin-LR-induced cytogenotoxicity. Free Radic. Res., 2007, 41 (12), 1326-1337.

[38] Pichardo, S.; Jos, A.; Zurita, J.L.; Salguero, M.; Cameán, A.M.; Repetto, G. Acute and subacute toxic effects produced by microcystin-YR on the fish cell lines RTG-2 and PLHC-1. Toxicol. In Vitro, 2007, 21, 1460-1467.

[39] Sicińska, P.; Bukowska, B.; Michałowicz, J.; Duda, W. Damage of cell membrane and antioxidative system in human erythrocytes incubated with microcystin-LR in vitro. Toxicon, 2006, 47, 387-397.

[40] Guzman, R.E.; Solter, P.F. Hepatic oxidative stress following prolonged sublethal microcystin LR exposure. Toxicol. Pathol., 1999, 27 (5), $582-$ 588.

[41] Weng, D.; Lu, Y.; Wei, Y.; Liu, Y.; Shen, P. The role of ROS in microcystin-LR-induced hepatocyte apoptosis and liver injury in mice. Toxicology, 2007, $232(1-2), 15-23$.

[42] Qiu, T.; Xie, P.; Liu, Y.; Li, G.; Xiong, Q.; Hao, L.; Li, H. The profound effects of microcystin on cardiac antioxidant enzymes, mitochondrial function and cardiac toxicity in rat. Toxicology 2009, 257 (1-2), 86-94.

[43] Botha, N.; Gehringer, M. M.; Downing, T.G.; Van De Venter, M.; Shephard, E.G. The role of microcystin-LR in the induction of apoptosis and oxidative stress in Caco-2 cells. Toxicon, 2004, 43 (1), 85-92.

[44] Ding, W.X.; Shen, H.M.; Ong, C.N. Pivotal role of mitochondrial $\mathrm{Ca}\left({ }^{2+}\right)$ in microcystin-induced mitochondrial permeability transition in rat hepatocytes. Biochem. Biophys. Res. Commun., 2001, 285 (5), 1155-1161.

[45] Zhang, H.; Zhang, J.; Chen, Y.; Zhu, Y. Microcystin-RR induces apoptosis in fish lymphocytes by generating reactive oxygen species and causing mitochondrial damage. Fish Physiol. Biochem., 2008, 34 (4), 307-312.

[46] Wei, Y.; Weng, D.; Li, F.; Zou, X.; Young, D.O.; Ji, J.; Shen, P. Involvement of JNK regulation in oxidative stress-mediated murine liver injury by microcystin-LR. Apoptosis, 2008, 13 (8), 1031-1042.

[47] Puerto, M.; Pichardo, S.; Jos, Á.; Prieto, A. I.; Sevilla, E.; Frías, J. E.; Cameán, A.M. Differential oxidative stress responses to pure Microcystin-LR and Microcystin-containing and non-containing cyanobacterial crude extracts on Caco-2 cells. Toxicon, 2010, 55, 514-522.

[48] Huang, X.; Chen, L.; Liu, W.; Qiao, Q.; Wu, K.; Wen, J.; Huang, C.; Tang, R.; Zhang, X. Involvement of oxidative stress and cytoskeletal disruption in microcystin-induced apoptosis in CIK cells. Aquat. Toxicol., 2015, 165, 41-50.

[49] Meng, G.; Liu, J.; Lin, S.; Guo, Z.; Xu, L. Microcystin-LR-caused ROS generation involved in p38 activation and tau hyperphosphorylation in neuroendocrine (PC12) cells. Environm. Toxicol., 2013, 30(3):366-74. 
[50] Chen, Y.; Zhou, Y.; Wang, X.; Qian, W.; Han, X. Microcystin-LR induces autophagy and apoptosis in rat Sertoli cells in vitro. Toxicon, 2013, 76, 84-93.

[51] Huguet, A.; Henri, J.; Petitpas, M.; Hogeveen, K.; Fessard, V. Comparative cytotoxicity, oxidative stress, and cytokine secretion induced by two cyanotoxin variants, microcystin LR and RR, in human intestinal Caco-2 cells. J. Biochem. Mol. Toxicol., 2013, 27 (5), $253-258$.

[52] Jiang, J.; Shan, Z.; Xu, W.; Wang, X.; Zhou, J.; Kong, D.; Xu, J. Microcystin-LR induced reactive oxygen species mediate cytoskeletal disruption and apoptosis of hepatocytes in Cyprinus carpio L. PLoS One, 2013, 8 (12), 1-10.

[53] Lu, Y.-F.; Liu, J.; Wu, K. C.; Qu, Q.; Fan, F.; Klaassen, C.D. Overexpression of Nrf2 protects against microcystin-induced hepatotoxicity in mice. PLoS One, 2014, 9 (3), e93013.

[54] Itoh, K.; Wakabayashi, N.; Katoh, Y.; Ishii, T.; Igarashi, K.; Engel, J.D.; Yamamoto, M. Keap1 represses nuclear activation of antioxidant responsive elements by Nrf2 through binding to the amino-terminal Neh2 domain. Genes Dev., 1999, 13 (1), 76-86.

[55] Yamamoto, T.; Suzuki, T.; Kobayashi, A.; Wakabayashi, J.; Maher, J.; Motohashi, H.; Yamamoto, M. Physiological significance of reactive cysteine residues of Keap1 in determining Nrf2 activity. Mol. Cell. Biol., 2008, 28 (8), 2758-2770.

[56] Ohta, T.; Nishiwaki, R.; Yatsunami, J.; Komori, A.; Suganuma, M.; Fujiki, H. Hyperphosphorylation of cytokeratins 8 and 18 by microcystin-LR, a new liver tumor promoter, in primary cultured rat hepatocytes . Carcinogenesis, 1992, 13 (12 ), 2443-2447.

[57] Gácsi, M.; Antal, O.; Vasas, G.; Máthé, C.; Borbély, G.; Saker, M.L.; Gyori, J.; Farkas, A.; Vehovszky, A.; Bánfalvi, G. Comparative study of cyanotoxins affecting cytoskeletal and chromatin structures in CHO-K1 cells. Toxicol. In Vitro, 2009, 23 (4), 710-718.

[58] Komatsu, M.; Furukawa, T.; Ikeda, R.; Takumi, S.; Nong, Q.; Aoyama, K.; Akiyama, S. I.; Keppler, D.; Takeuchi, T. Involvement of mitogenactivated protein kinase signaling pathways in microcystin-LR-Induced apoptosis after its selective uptake mediated by OATP1B1 and OATP1B3. Toxicol. Sci., 2007, 97 (2), 407-416.

[59] Zeng, J.; Tu, W.; Lazar, L.; Chen, D.; Zhao, J.; Xu, J. Hyperphosphorylation of microfilament-associated proteins is involved in microcystin-LRinduced toxicity in HL7702 cells. Environ. Toxicol., 2015, 30 (8), 981-988.

[60] Chen, D.N.; Zeng, J.; Wang, F.; Zheng, W.; Tu, W.W.; Zhao, J.S.; Xu, J. Hyperphosphorylation of intermediate filament proteins is involved in microcystin-LR-induced toxicity in HL7702 cells. Toxicol. Lett., 2012, 214 (2), 192-199.

[61] Feng, G.; Abdalla, M.; Li, Y.; Bai, Y. NF-кB mediates the induction of Fas receptor and Fas ligand by microcystin-LR in HepG2 cells. Mol. Cell. Biochem., 2011, 352 (1-2), 209-219.

[62] Gilmore, T.D. The Rel/NF-kappaB signal transduction pathway: introduction. Oncogene, 1999, 18 (49), $6842-6844$.

[63] Ji, Y.; Lu, G.; Chen, G.; Huang, B.; Zhang, X.; Shen, K.; Wu, S. Microcystin-LR induces apoptosis via NF-kB /iNOS pathway in INS-1 cells. Int. J. Mol. Sci., 2011, 12 (7), 4722-4734.

[64] Vesterkvist, P.S.M.; Misiorek, J.O.; Spoof, L.E.M.; Toivola, D.M.; Meriluoto, J.A.O. Comparative cellular toxicity of hydrophilic and hydrophobic microcystins on Caco-2 cells. Toxins (Basel), 2012, 4 (11), 1008-1023.

[65] Rymuszka, A.; Adaszek, Ł. Cytotoxic effects and changes in cytokine gene expression induced by microcystin-containing extract in fish immune cells-an in vitro and in vivo study. Fish Shellfish Immunol., 2013, 34 (6), 1524-1532.

[66] Krakstad, C.; Herfindal, L.; Gjertsen, B.T.; Bøe, R.; Vintermyr, O.K.; Fladmark, K.E.; Døskeland, S.O. CaM-kinaseII-dependent commitment to microcystin-induced apoptosis is coupled to cell budding, but not to shrinkage or chromatin hypercondensation. Cell Death Differ., 2006, 13 (7), $1191-1202$.

[67] Li, T.; Ying, L.; Wang, H.; Li, N.; Fu, W.; Guo, Z.; Xu, L. Microcystin-LR induces ceramide to regulate PP2A and destabilize cytoskeleton in HEK293 cells. Toxicol Sci., 2012, 128(1), 147-57.

[68] Hanson, C.J.; Bootman, M.D.; Distelhorst, C.W.; Maraldi, T.; Roderick, H.L. The cellular concentration of Bcl-2 determines its pro- or antiapoptotic effect. Cell Calcium, 2008, 44 (3), 243-258.

[69] Hoof, C. Van; Goris, J. Phosphatases in apoptosis: to be or not to be, PP2A is in the heart of the question. Biochim. Biophys. Acta - Mol. Cell Res., 2003, 1640 (2-3), 97-104.

[70] Dobrowsky, R.T.; Kamibayashi, C.; Mumby, M.C.; Hannun, Y.A. Ceramide activates heterotrimeric protein phosphatase 2A. J. Biol. Chem., 1993, $268(21), 15523-15530$.

[71] Galadari, S.; Kishikawa, K.; Kamibayashi, C.; Mumby, M.C.; Hannun, Y.A. Purification and characterization of ceramide-activated protein phosphatases. Biochemistry, 1998, 37 (32), 11232-11238.

[72] Wang, X.; Huang, P.; Liu, Y.; Du, H.; Wang, X.; Wang, M.; Wang, Y.; Hei, T. K.; Wu, L.; Xu, A. Role of nitric oxide in the genotoxic response to chronic microcystin-LR exposure in human-hamster hybrid cells. J. Environ. Sci. (China), 2015, 29, 210-218.

[73] Kist, L.W.; Rosemberg, D.B.; Pereira, T.C.B.; De Azevedo, M.B.; Richetti, S.K.; De Castro Leão, J.; Yunes, J.S.; Bonan, C.D.; Bogo, M.R. Microcystin-LR acute exposure increases AChE activity via transcriptional ache activation in zebrafish (Danio rerio) brain. Comp. Biochem Physiol. - C Toxicol. Pharmacol., 2012, 155 (2), 247-252.

[74] Meng, G.; Sun, Y.; Fu, W.; Guo, Z.; Xu, L. Microcystin-LR induces cytoskeleton system reorganization through hyperphosphorylation of tau and HSP27 via PP2A inhibition and subsequent activation of the p38 MAPK signaling pathway in neuroendocrine (PC12) cells. Toxicology, 2011, 290 (2-3), 218-229.

[75] Ballatore, C.; Lee, V.M.-Y.; Trojanowski, J.Q. Tau-mediated neurodegeneration in Alzheimer's disease and related disorders. Nat. Rev. Neurosci., 2007, $8(9), 663-672$. 
[76] Li, G.; Cai, F.; Yan, W.; Li, C.; Wang, J. A proteomic analysis of MCLR-induced neurotoxicity: implications for Alzheimer's disease. Toxicol. Sci., 2012, 127 (2), 485-495.

[77] Ding, X.-S.; Li, X.-Y.; Duan, H.-Y.; Chung, I.-K.; Lee, J.-A. Toxic effects of Microcystis cell extracts on the reproductive system of male mice. Toxicon, 2006, 48 (8), 973-979.

[78] Li, Y.; Sheng, J.; Sha, J.; Han, X. The toxic effects of microcystin-LR on the reproductive system of male rats in vivo and in vitro. Reprod. Toxicol., 2008, 26 (3-4), 239-245.

[79] Chen, L.; Zhang, X.; Zhou, W.; Qiao, Q.; Liang, H.; Li, G.; Wang, J.; Cai, F. The interactive effects of cytoskeleton disruption and mitochondria dysfunction lead to reproductive toxicity induced by microcystin-LR. PLoS One, 2013, 8 (1), e53949.

[80] Zhang, H.-Z.; Zhang, F.-Q.; Li, C.-F.; Yi, D.; Fu, X.-L.; Cui, L.-X. A cyanobacterial toxin, microcystin-LR, induces apoptosis of sertoli cells by changing the expression levels of apoptosis-related proteins. Tohoku J. Exp. Med., 2011, 224 (3), 235-242.

[81] Zhou, Y.; Chen, Y.; Yuan, M.; Xiang, Z.; Han, X. In vivo study on the effects of microcystin-LR on the apoptosis, proliferation and differentiation of rat testicular spermatogenic cells of male rats injected i.p. with toxins. J. Toxicol. Sci., 2013, 38 (5), 661-670.

[82] Wu, J.; Shao, S.; Zhou, F.; Wen, S.; Chen, F.; Han, X. Reproductive toxicity on female mice induced by microcystin-LR. Environ. Toxicol. Pharmacol., 2014, 37 (1), 1-6.

[83] Humpage, A.R.; Falconer, I.R. Microcystin-LR and liver tumor promotion: Effects on cytokinesis, ploidy, and apoptosis in cultured hepatocytes. Environ. Toxicol., 1999, 14 (1), 61-75.

[84] IARC. IARC monographs on the evaluation of carcinogenic risks to humans. Ingested nitrate and nitrite, and cyanobacterial peptide toxins. IARC Monogr. Eval. Carcinog. Risks Hum., 2010, 94( v - vii), 1-412.

[85] Clark, S.P.; Ryan, T.P.; Searfoss, G.H.; Davis, M.A.; Hooser, S.B., Chronic microcystin exposure induces hepatocyte proliferation with increased expression of mitotic and cyclin-associated genes in P53-deficient Mice. Toxicol. Pathol., 2008, 36 (2), 190-203.

[86] Dias, E.; Matos, P.; Pereira, P.; Batoréu, M.C.C.; Silva, M.J.; Jordan, P. Microcystin-LR activates the ERK1/2 kinases and stimulates the proliferation of the monkey kidney-derived cell line Vero-E6. Toxicol. In Vitro, 2010, 24 (6), 1689-1695.

[87] Li, H.; Xie, P.; Li, G.; Hao, L.; Xiong, Q. In vivo study on the effects of microcystin extracts on the expression profiles of proto-oncogenes (c-fos, cjun and c-myc) in liver, kidney and testis of male Wistar rats injected i.v. with toxins. Toxicon, 2009, 53 (1), 169-175.

[88] Fan, H.; Cai, Y.; Xie, P.; Xiao, W.; Chen, J.; Ji, W.; Zhao, S. Microcystin-LR stabilizes c-myc protein by inhibiting protein phosphatase 2A in HEK293 cells. Toxicology, 2014, 319 (1), 69-74.

[89] Fu, W.; Yu, Y.; Xu, L. Identification of temporal differentially expressed protein responses to microcystin in human amniotic epithelial cells. Chem. Res. Toxicol., 2009, 22 (1), 41-51.

[90] Takumi, S.; Komatsu, M.; Furukawa, T.; Ikeda, R.; Sumizawa, T.; Akenaga, H.; Maeda, Y.; Aoyama, K.; Arizono, K.; Ando, S.; Takeuchi, T. p53 plays an important role in cell fate determination after exposure to microcystin-LR. Environ. Health Persp., 2010, 118 (9), $1292-1298$. 


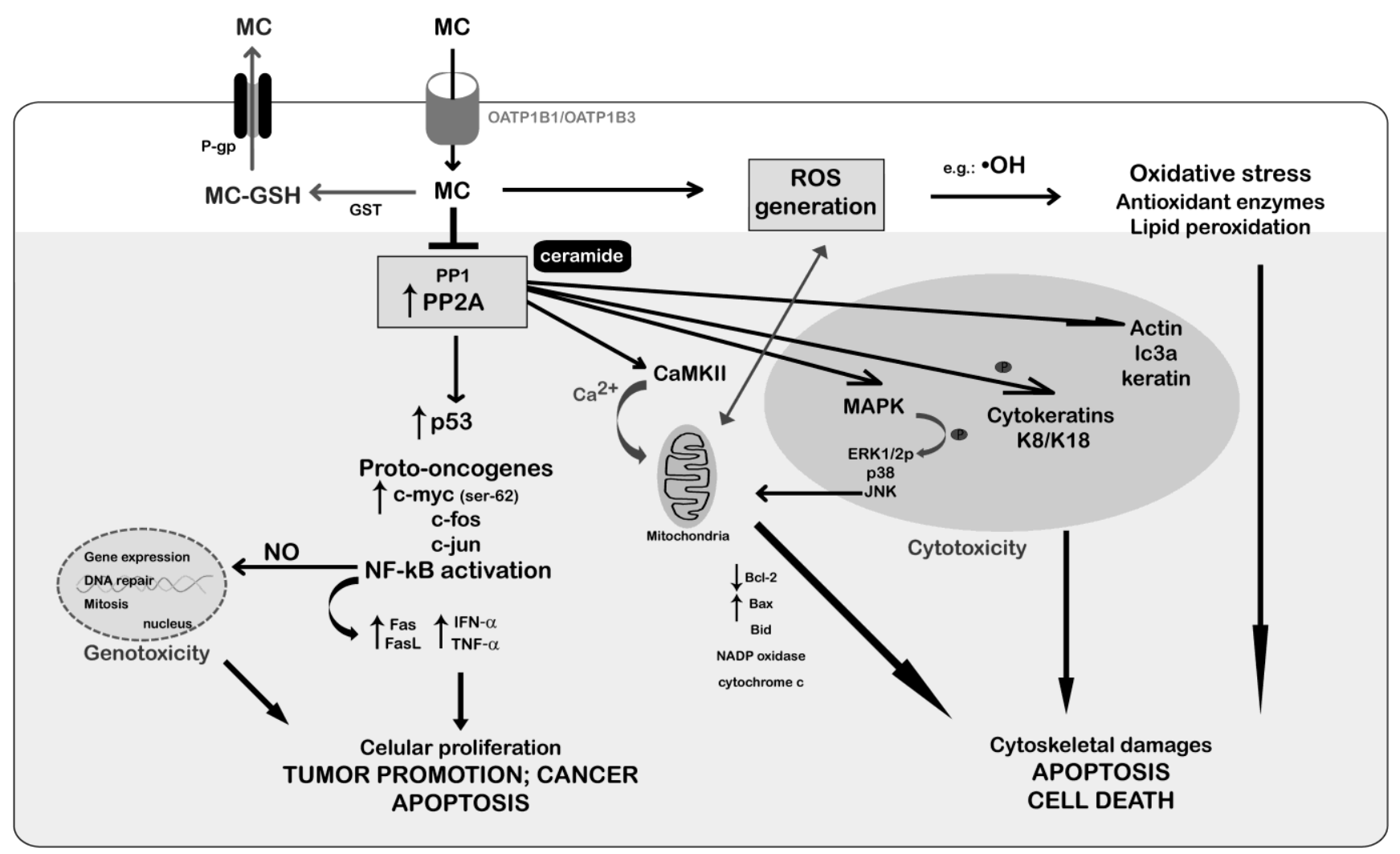

Figure 1. Proposed pathways of MCs uptake, effects and mechanisms of toxicity, biotransformation and elimination on animal cells, to summarize the so far described MC toxicity mechanisms. Legend: CaMKII (calcium calmodulin-dependent multifunctional protein kinase II); Ceramide = second messenger; c-fos, c-jun; c-myc (transcription factors); ERK1/2 (extracellular-signal-regulated kinase); GSH (reduced glutathione); GST (Glutathione S-transferase); JNK (c-Jun N-terminal protein kinase); MAPK (Mitogen-activated protein kinase); NF-кB (nuclear factor kappa-light-chain-enhancer of activated B cells); OATP (organic anion transporter polypeptide); P (phosphate group); P-gp (P-glycoprotein); PP1/PP2A (protein phosphatases PPI and PP2A); ROS (reactive oxygen species); $\bullet \mathrm{OH}$ (hydroxyl radical); NO (nitric oxide); $\uparrow$ or $\downarrow$ refer to changes in expression, $\perp$ refers to inhibition. 